\title{
Correction to: Patterns and predictors of registration and participation at a supportive care program for prostate cancer survivors
}

\author{
Lindsay Hedden ${ }^{1,2,3} \cdot$ Phil Pollock $^{2} \cdot$ Stirling Bryan ${ }^{1,3} \cdot$ Larry Goldenberg $^{2,4} \cdot$ Celestia Higano $^{2,4,5}$
}

Published online: 16 July 2019

(C) Springer-Verlag GmbH Germany, part of Springer Nature 2019

\section{Correction to: Supportive Care in Cancer}

https://doi.org/10.1007/s00520-019-04927-6

The name of Stirling Bryan was incorrectly captured in the original manuscript and is now corrected in this article.

Publisher's note Springer Nature remains neutral with regard to jurisdictional claims in published maps and institutional affiliations.

The online version of the original article can be found at https://doi.org/ 10.1007/s00520-019-04927-6

Lindsay Hedden

Lindsay.hedden@ubc.ca

1 School of Population and Public Health, University of British Columbia, 2206 E Mall, Vancouver, BC V6T 1Z3, Canada

2 Vancouver Prostate Centre, 2660 Oak St, Vancouver, BC V6H 3Z6, Canada

3 Centre for Clinical Epidemiology and Evaluation, 7th Floor, 828 West 10th Avenue, Vancouver, BC V5Z 1M9, Canada

4 Department of Urologic Sciences, University British Columbia, 6th Floor, 2775 Laurel Street, Vancouver, BC V5Z 1M9, Canada

5 Fred Hutchinson Cancer Research Centre, University ofWashington, 1100 Fairview Ave N, Seattle, WA, USA 